\title{
Illustrating the Gaps and Needs in the Training Support of Community Health Workers in India
}

\author{
Deepika Yadav \\ IIIT-Delhi \\ New Delhi, India \\ deepikay@iiitd.ac.in \\ Kirti Dabas \\ IIIT-Delhi \\ New Delhi, India \\ kirtid@iiitd.ac.in
}

\author{
Prerna Malik \\ IIIT-Delhi \\ New Delhi, India \\ prernam@iiitd.ac.in \\ Pushpendra Singh* \\ IIIT-Delhi \\ New Delhi, India \\ psingh@iiitd.ac.in
}

\begin{abstract}
In India and other developing countries, Community Health Workers (CHWs) provide the first line of care in delivering necessary maternal and child health services. In this work, we assess the training and skill-building needs of CHWs, through a mobile-based training intervention deployed for six months to $500 \mathrm{CHWs}$ for conducting 144 training sessions in rural India. We qualitatively probed 1178 questions, asked by CHWs, during training sessions, and conducted a content analysis of the learning material provided to CHWs. Further, we interviewed $48 \mathrm{CHWs}$ to understand the rationale of information seeking and perceptions of training needs. We present our understanding of the knowledge gaps of CHWs and how the current learning material and training methods are ineffective in addressing it. Our study presents design implications for HCI4D researchers for mobile learning platforms targeted towards $\mathrm{CHWs}$. We also provide policy-level suggestions to improve the training of CHWs in India or a similar context.
\end{abstract}

\section{CCS CONCEPTS}

- Human-centered computing $\rightarrow$ Human computer interaction (HCI); Empirical studies in HCI;

\section{KEYWORDS}

CHW, ASHA, mHealth, HCI4D, ICTD

\section{ACM Reference Format:}

Deepika Yadav, Prerna Malik, Kirti Dabas, and Pushpendra Singh. 2021. Illustrating the Gaps and Needs in the Training Support of Community Health Workers in India. In CHI Conference on Human Factors in Computing Systems (CHI '21), May 8-13, 2021, Yokohama, Japan. ACM, New York, NY, USA, 16 pages. https://doi.org/10.1145/3411764.3445111

*corresponding author

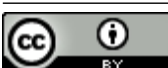

This work is licensed under a Creative Commons Attribution International 4.0 License.

CHI '21, May 8-13, 2021, Yokohama, Japan

(c) 2021 Copyright held by the owner/author(s).

ACM ISBN 978-1-4503-8096-6/21/05.

https://doi.org/10.1145/3411764.3445111

\section{INTRODUCTION}

Acute shortages of health professionals and the increasing burden of diseases in the Global South have renewed focus on community health workers (CHWs) [103]. CHWs are increasingly being recognized to play a significant role in extending services to "hard to reach' groups and areas and substituting health professionals for a range of tasks [103]. CHWs are defined as "health workers carrying out functions related to healthcare delivery; specifically trained in the context of the intervention, and having no formal professional or paraprofessional certificate in tertiary education" $[49,78]$. Being from the communities CHWs serve enables them to act as a liaison/intermediary between the health system and the community to facilitate access to health services and promote health [31, 37, 74].

Evidence supports the significant contribution of CHWs in delivering essential health interventions, particularly for maternal and child health [49]. However, their sub-optimal performance remains a concern. Systematic reviews studying the factors influencing the performance of $\mathrm{CHWs}$ have highlighted training and supervision as one of the most neglected intervention-related factor [42]. Within $\mathrm{HCI}, \mathrm{CSCW}$, and ICTD communities also, thus far, the focus tends to be towards improving the performance by investigating solutions for data collection, health education, adherence to clinical protocols, workflows and communication [48, 60, 83, 85]. There is a paucity of research on the training and education of CHWs. Few works in academic settings have attempted to address the problem of improving knowledge and skills of CHWs using technology as a medium [36, 106, 108, 109]. We contribute to this space through a longitudinal study of deploying a mobile-based training solution for 500 CHWs in India. We extend our prior work [109] in which we proposed a low-cost, mobile-based training system for CHWs in a similar Indian context. While that limited to understanding the tool's efficacy, in this study, we are using the tool to understand the training gaps, needs, and aspirations of CHWs by conducting a longitudinal study.

Over a period of six months, $500 \mathrm{CHWs}$ were trained on six topics of Home-Based Post Natal Care in 20 batches. We focused on studying the information seeking of $\mathrm{CHWs}$ by analyzing their queries. Through 144 training sessions, composed of delivering audio lectures on the training topics and facilitating interaction between an expert and CHWs, we collected 1178 questions. We 
examined the questions both qualitatively and quantitatively to find the topics and the nature of information seeking. We also mapped the coverage of these questions with the reference material of CHWs and collected CHWs' experiences by interviewing 48 of them.

We observed that when CHWs received the opportunity of interacting with an expert, they sought knowledge on field cases to improve problem-solving skills. The three key broad themes for improvement were routine patient care, knowledge on identifying danger signs, and skills to support breastfeeding. The findings highlighted the need to improve on-the-job support system. We looked at the need to adopt holistic approaches such as improving the coverage and presentation of reference material, enhancing the training structure for problem-solving, and providing forums for interaction with experts.

The contributions of our study are primarily two. First, we present the first longitudinal study in the HCI4D literature that goes deep into examining the knowledge gaps of CHWs by studying information seeking of 500 workers over six months. We complement prior studies in this domain by collecting day to day field queries of CHWs and understanding what kind of knowledge CHWs seek to address them. Second, we contribute to the HCI4D community, which has been increasingly growing around CHWs, maternal and child health contexts in disadvantaged groups by outlining key takeaways for designing holistic learning solutions targeted towards health education of CHWs.

\section{BACKGROUND}

India is the second-most populous country in the world. There are 1.2 billion people, and $70 \%$ of them live in rural areas [70]. Public health challenges are in huge volumes and have a worse impact on rural populations. According to the National Family Health Survey-4, the gap in the decline of under-five mortality rates (U5MR) between the richest and the poorest is three-fold, with 23 per 1000 live birth in the highest wealth quintile to 72 deaths per 1000 live births in the lowest wealth quintile [73]. The rural areas have a greater number of under-five deaths, 56 deaths per 1,000 live births as compared to 34 deaths per 1,000 live births in urban areas.

Further, the acute shortages of health workers and their uneven distribution make healthcare challenging to access. According to Rural Health Statistics, the density of doctors, nurses, and midwives in urban areas (13.9 and 15.1 per 10000 population) are three times higher than rural areas (3.9 and 4.1 per 10,000 population) [72].

To improve healthcare access to the rural population, particularly women, the Indian government took a key initiative in 2005 by reviving its community health worker program. A new cadre of community health workers known as Accredited Social Health Activist (ASHA) was created to improve the link between primary healthcare and the communities [55, 57]. Every village, with an approximate population of 1000 , has been equipped with an ASHA- a woman selected from within the village [55]. At present, close to a million (979826) ASHAs are appointed across the country $[56,65]$.

\section{RELATED WORK}

Our research contributes to a growing body of HCI4D literature that focuses on mobile technology for development in under-served communities and mHealth research on maternal health and CHWs.

\subsection{Technology for Development}

Reducing cost of ICTs, particularly of mobile phones, has given momentum to HCI4D research that examines the intersection of computing technologies and sustainable development in low-income settings [22]. There is increasing awareness in the HCI community, albeit slow, about the distinct challenges the populations in Global South face while interacting with technology-mediated solutions [47]. For example, how the infrastructural limitations, literacy barriers, social and institutional problems make the implementation of even the most basic computing solutions challenging $[11,35,54]$.

3.1.1 Voice Platforms. Voice-based forums, which allow addressing the constraints of literacy, language, and Internet access, have emerged as a viable medium for information exchange in these settings. There is a strong body of work that has designed, deployed, and evaluated the Interactive Voice Response (IVR) systems for a variety of applications [59, 63, 101]. An early work, SpokenWeb envisioned and pioneered a telecom version of Web to allow illiterate and under-privileged users create and make use of the Web through voice-based operations [45]. The field experiments, in particular, demonstrated the usability of voice message boards, which paved the way for further research in this direction. Patel et al. extended the idea by developing an interactive voice forum for farmers, Avaaj Otalo [84]. The seminal work studied the use of online communities (in form of IVR-based voice forum) for the rural population and found promising results in terms of users leveraging the platform for interacting with the experts and peers in a structured manner. Such systems continue to create impact in under-resourced regions covering purposes such as social inclusion [29], citizen journalism [63], medication adherence [19] and dissemination of public health information [2].

3.1.2 Learning Technologies for Developing Countries. As computers ventured into developing countries, there was widespread enthusiasm to revolutionize education in low-resource settings. Many ambitious, technocentric initiatives were laid to equip rural schools with computers, e.g., One Laptop per Child $[6,18]$, Hole in the Wall [58], etc. However, there is no concrete evidence about how putting off-the-shelf computing device into learners' hand help them. Furthermore, the projects did not attain success [44]. As discussed by Toyoma and others, many fundamental problems, e.g., lack of infrastructure, the motivation of teachers, socio-cultural barriers, parents unawareness, child labor, need attention [93, 94].

Lack of quality teaching, which has been a persistent problem in both K-12 and higher education in India [20] has been tried to be addressed by several interventions by bringing expertise from outside through connecting local teachers with experts in developed countries $[8,91]$ and investigating pedagogies to adapt and utilize global online education content, e.g., MOOCs [20]. 
Mobile phones offer the convenience of expanding learning opportunities to more informal forums and to a wide range of learners. Educational technologies deployed in low-resource communities have primarily concentrated on students and teachers. Kam et al. performed multiple studies designing game-based applications for language learning of rural children $[39,46]$. Several recent studies have focused on using e-readers, tablets, and smartphones in the classroom and after-school environments $[88,97,98]$ and how teachers perspective should be considered in designing technologies [99, 100]. As discussed earlier and highlighted by Poon et al. in the educational context, feature phones are still the most preferred choice [86]. Moreover, we are far from a good understanding of how we might design effective educational interventions over inexpensive but more ubiquitous feature phones. Particularly for the workers and para-professionals such as $\mathrm{CHWs}$ who need in-service and continued opportunities for learning. Madaio et al. deployed an IVR literacy technology for children in 8 rural communities of Côte d'Ivoire for several months and provided deep insights into how families viewed their learning goals and looked for technology supporters, and transitioned into autonomous supervisory modes [51]. Such long-term deployments reveal many new aspects that are important for future designs. On a similar line, we attempt to bring out the learning needs through a longitudinal study. We will discuss specifically what has been done in the educational context of CHWs in the following subsections.

\subsection{Technology and Maternal Health}

Since recent years, attention has grown towards problems concerning women and maternal health. Different aspects of care have been highlighted, ranging from motherhood challenges [12] to menstrual health $[15,96]$ to intimate care $[4,5]$ and how and in what forms women seek support from digital platforms [7, 26, 30]. Works by Fourney et al. [26] and Gui et al. [30], which are closer to ours investigated information-seeking patterns of women on the Internet and online communities; informing about the temporal characteristics of queries and typology of searches. However, most of these belong to developed settings.

In underserved settings, prior work focuses on improving adherence to treatments [81], health education [48, 87], and health information services [85]. Mustafa et al. revealed the challenges in implementing maternal health care interventions in patriarchal contexts relating to ingrained beliefs in traditional practices and lack of autonomy of women [64]. Some of the notable works in these regions has been conducted as part of mHealth initiatives. These primarily aim to improve antenatal and maternal health by addressing existing inefficiencies in the health systems. For example, MomConnect is a flagship program of the South African National Department of Health [13] that delivers stage-based targeted health information to pregnant and postpartum women. It is the largest mHealth initiative in the world. Presently, MomConnect has over one and a half million end-users. The central technology for delivering information is SMS. Registered women receive weekly messages based on their stage of pregnancy. Similar to this, other examples are Kilkari [1] and mMitra in India (IVR-based calling system) [9], and Aponjon in Bangladesh (SMS-based) [3].

\subsection{Technology and CHWs}

Derenzi et al. identify six essential health functions where mobile applications have been designed for CHWs: data collection, communication between health workers, job aids and decision support, health workers' supervision, health promotion, training, and access to reference material [23]. Some of the routine data collection activities that $\mathrm{CHWs}$ perform include household surveys, routine reporting, and supervisory reports [23]. These are mostly done manually through the paper, which incurs inefficiency and errors. A variety of efforts have been made in digitizing the process, ranging from using PDAs and mobile apps to hybrid systems such as CAM, ODK Scan $[21,83]$. However, going paperless is challenging due to workplace practice change, lack of comfort with digital devices, low education levels, and the complexities of contexts in which data collection activities are performed [34, 82]

To improve timeliness of CHWs' home visits and communication between $\mathrm{CHWs}$ and supervisors, researchers have examined text messaging extensively [24, 111] alongwith methods to improve the feedback loop by allowing CHWs see their performance data [25] . E-checklist has been explored to help decision making and adherence to clinical protocols of patient examination. Further, mobile videos have emerged to be an effective form of job aid for assisting CHWs in client counseling [48, 60, 61, 87].

However, few investigations directly aim to study the role of technology in the training and education of CHWs. The area is underexplored. The majority of the contribution comes from mHealth projects. These applications commonly address the access to information and provision of supplementary training (e.g., refresher training courses). For example, the Millennium Village Project in Uganda facilitates continuing education to $\mathrm{CHWs}$ by making training modules available in mobile file formats [16]. CHWs can reinforce their learning by reading the modules, taking tests, and receiving immediate feedback. TulaSalud in Guatemala provides distance education through the digital learning material and teleconferencing to community auxiliary nurses [95]. Mobile Academy in India aims to upgrade CHWs knowledge on healthy life-saving behaviors through an IVR-based audio training course [27], and CapacityPlus project deliver refresher training in spaced education format in Senegal [33] through an IVR-based mLearning system. We attempted to fill the gap by proposing a multi-purpose training platform in our prior work $[106,109]$. The system uses telephony (IVR) at its core that makes it suitable for other resource-constrained settings. The corresponding studies have shown positive gains in terms of knowledge gain and peer-to-peer learning effects. We extend our work by using the system to conduct a longitudinal study and uncover the information-seeking patterns of CHWs.

\section{METHODOLOGY}

\subsection{Training System}

For the implementation, we used an existing, publicly available training system proposed by us in a prior work [109]. Featurewise, the system supported two of our essential needs. First, the system was affordable, easy to set up, did not impose the need to procure any additional digital device. Running the system required a single smartphone, a computer, an internet connection on the trainer's side, and access to basic feature phones on the ASHAs' side. 
All ASHAs have access to personal phones and receive official SIM cards. Second, the system facilitates hosting online training sessions, thus avoiding the travel overhead for ASHAs and supporting live interaction between the ASHAs and the trainers at the same time.

The system adapts usual cellular conference calls to facilitate systematic interaction between trainers and participants. There are two interfaces for trainers - a web portal for designing and scheduling training sessions and an android app to host sessions in real-time. The participants, on the other hand, interacts through IVR mediated voice calls. In a connected call, the trainer plays pre-recorded audio material and monitors and controls trainees' speaking. The participants remain muted all the time except when they want to speak. If a participant wants to speak, The presses ' 1 ' on their phone to request access to speak. The system captures the generated DTMF code, and it is shown as a 'raised hand' icon on the trainer's smartphone. For more details of the system architecture, we recommend reading $[106,109]$.

\subsection{Participants}

We partnered with an NGO working in the rural areas of a North Indian state, Haryana. The NGO works in partnership with the state government organization - National Health Mission - and has been assigned ASHAs of two districts, which includes 1648 ASHAs covering a population of 2.2 million. We selected ASHAs using convenience sampling. The NGO staff first connected with their points of contacts at the community health center levels (CHCs, a health facility at tier-3). The in-charge officials at $\mathrm{CHCs}$ helped reach out to ASHAs by introducing the proposed training intervention and recruiting ASHAs. Based on ASHAs' interest and availability, the ASHAs were included till we reached our sample size of 500 . We obtained ASHAs consent by soliciting written notes sent to the NGO staff as WhatsApp messages or simple text messages. Our selection process resulted in coverage of $10 / 10 \mathrm{CHCs}$ with an average number of 50 ASHAs per $\mathrm{CHC}$ (minimum was six from two $\mathrm{CHCs}$, the maximum was 100 from one $\mathrm{CHC}$ ). The participants' demographic details are given in the table 1. All participants spoke Hindi with accents of Haryana and Punjab regions. Participants were remunerated for their time by cash incentive; each ASHA received an amount of $1000 \mathrm{INR}$.

\subsection{Intervention Execution}

The planning and preparation phase of the intervention started six months before the actual implementation. Our NGO partner was situated $244 \mathrm{~km}$ away from our institute. Several face to face meetings were organized to understand the resource availability, budget, and methodology. After developing a high level of initial intervention design, we began setting up the training system, which included adjusting the code to suit our requirements (adding functionalities in the web portal and android app), configuring the server and telephony gateway.

Two of the authors made back and forth visits to the NGO to conduct training workshops and facilitate running mock training sessions. With ten team members involved (7 from the NGO staff), 12 days were devoted to the testing period, and 18 mock training sessions were conducted. Initially, few sessions were dedicated to test the functions of the android app and the web portal, which
Table 1: Participants Demographics

\begin{tabular}{lr}
\hline Parameters & Number of ASHAs(\%) \\
\hline Age & $20(4 \%)$ \\
$<30$ & $242(48 \%)$ \\
$30-40$ & $188(38 \%)$ \\
$40-50$ & $28(6 \%)$ \\
$>50$ & $22(4 \%)$ \\
no information & \\
Education (grade) & \\
$5^{\text {th }}-8^{\text {th }}$ & $12(2 \%)$ \\
$8^{\text {th }}-10^{\text {th }}$ & $79(16 \%)$ \\
$10^{\text {th }}-12^{\text {th }}$ & $357(72 \%)$ \\
Graduation and above & $30(6 \%)$ \\
no information & $22(4 \%)$ \\
Experience (years) & \\
$0-5$ & $21(4 \%))$ \\
$5-10$ & $201(40 \%)$ \\
$10-15$ & $222(45 \%)$ \\
$>15$ & $34(7 \%)$ \\
no information & $22(4 \%)$ \\
\hline
\end{tabular}

took approximately 8-10 minutes per session, followed by conducting full-length sessions of 20-30 minutes. Various kinds of issues with the system connectivity and functionality surfaced, e.g., network delays affecting the app when using mobile data, and were addressed.

For the final deployment, a team of eight team members - three authors from our side and five from our NGO partner. The NGO head, a pediatrician doctor with experience of 40 years, assumed the lead trainer's role, two staff members played the role of training moderators, and the rest acted as assistants to these moderators. We handled the responsibility of providing technical assistance to the NGO staff and overseeing the overall execution that included monitoring system logs, taking prompt action to rectify errors during live sessions, and maintaining data records.

For the training topic, we decided to choose the domain of HomeBased Postnatal Care (HBPNC) that aligns with the official training curriculum of ASHAs and has been identified as one of the most critical areas for improving the skills of ASHAs [52]. Under HBPNC, six topics were selected and prepared, namely breastfeeding, danger signs in mother, danger signs in newborn, newborn temperature control, play and communication with newborns, health, and mothers' happiness. For every topic, a 12-15 minute audio lecture was prepared by the doctor. The structure for a single training session composed of first delivering a topic content through the pre-recorded audio followed by question and answering with the doctor. Total seven sessions were planned for a batch - six topic sessions and one feedback session. Based on the findings of the prior studies [109], we decided the size of a training batch to be around 25. Using the sequential sampling method, we formed 20 groups consisting of about 25 ASHAs in each group. The session 
timing was fixed according to the ASHAs' availability. The majority of the ASHAs preferred time between 10:30 a.m and 1.00 p.m.

To manage the expert's availability, we modified our training design in the following manner. The training sessions were dedicated to delivering training content, conducting group discussions, and taking ASHAs questions, leaving out the live interaction with the doctor. Instead, the doctor's responses were collected offline. After finishing the training sessions of a day, the moderators would meet the doctor to record his responses. The moderator would then share these responses with the participants in the subsequent sessions.

This hybrid approach allowed us to use the expert's time efficiently. The lack of experts is an identified problem for training CHWs [41, 106, 107, 109].

We started our intervention in April 2019, completed it by August 2019. Throughout the deployment, we faced technical challenges that were navigated with technical intervention from our side. Some got resolved smoothly with modifications in the system code; for others, workarounds were developed by our team. However, many times, mainly due to slow internet speed, sessions faced frequent interruptions. We planned to conduct training sessions for two groups each working day. However, at times, we had to suspend the training sessions for a few days in some weeks. Also, due to availability issues of ASHAs, the batch size varied from 19 to 27 Even with prior notice of the training, some of the ASHAs had to go for other meetings and visits. In total, 144 sessions were conducted for the 20 groups, seven sessions per group. Three sessions were repeated for group 15 due to low attendance, and one was repeated for group 14. Overall, the average attendance of ASHAs was $76 \%$.

We obtained approval for the study from our institute's review board.

\subsection{Data Collection and Analysis}

Data collection was performed at various stages. The system server recorded every training session (audio recordings) and logged session event logs, e.g., call drops.

The training moderators and research staff made extensive field notes recording daily issues, observations, etc.

4.4.1 Q\&A Analysis. The ASHAs' questions and answers were extracted from the daily evening meetings' audio recordings between the moderators and the doctor. These were transcribed daily by one of the moderators. Two authors later transcribed the answers to the questions.

Both the authors were fluent in Hindi (the language of conversations) and English. The recordings were first transcribed into Hindi language and then translated into English. Since the native language of all the authors and the ASHAs was the same, we decided to perform the analysis on the Hindi transcriptions to understand the local terms and associated nuances better.

Three authors started the inductive thematic analysis of the questions individually by reading each question with two aspects in mind - the topics of the questions and the type of information seeking. After analyzing 112 questions, the authors discussed their codes and analysis rationale to develop an initial code dictionary.

Following this, two authors continued the analysis of the rest of the questions. After completing the annotation of every subsequent 200 questions, the authors discussed, refined, and added new emerged codes into the dictionary. While the codes for the type of information seeking saturated after the second iteration (eventually resulted in five themes), the codes for the topics and sub-topics increased until the last iteration. Finally, the final iteration reduced 30 codes of the main category to 15 and 321 sub-categories to 131 .

4.4.2 Content Analysis. A separate analysis activity was performed on the subset of the questions to understand the extent to which the reference material provided to ASHAs addresses them. We obtained e-material from the National Health Mission website, the apex government body managing the community health workers [57]. The material is divided into seven modules and serves both as the training and learning material. The modules are specifically organized in form of books provided to ASHAs when they get appointed and join the training program. We used these seven books along with an induction training module, which is a consolidated version of the first five modules for our analysis.

The two authors first understood the learning material by individually going through it and identified the relevant sections of the books to search content in. After this, the authors individually analyzed a few sample questions of two different topics to arrive at a consistent search and tagging method. For example, in addition to straightaway looking into the relevant chapters' sections, keyword searching at the individual file level and folder level was also used. While searching for a particular question topic, the authors also looked into related chapters that could potentially cover the topic indirectly, e.g., for a question related to consumption of iron supplement post-delivery was also checked in pregnancy care sections as both the stages are advised for iron consumption. Finally, all the questions of the selected topics (Home Based Postnatal Care, details in the finding section) were mapped to the extent of the coverage in terms of whether answers to the questions were present or not and if present then fully or partially.

4.4.3 Interviews. The last main activity of the study was interviewing the ASHAs. The aim was to collect ASHAs' training experiences, usability issues, feedback for future improvement, and general information on existing training mechanisms. The interview questions were of semi-structured form, e.g., "How many sessions you attended? If you missed some, then what were the reasons?" "Did you gain any benefit of the training in your work?", "What was the most useful feature of the system?".

The selection process of the participant followed a systematic sampling approach. We first shortlisted 100 ASHAs from the initial pool of 500 . From the 500 ASHAs already divided into 20 groups, we selected every 5 th member. Going serially, we obtained consent from 60 ASHAs and could successfully conduct interviews with 50. Two interviews were discarded from these due to a very low attendance rate (less than two sessions). The interviews were conducted telephonically by three authors in the Hindi language. On average, an interview took 30 minutes to complete. Interviews were recorded after obtaining the consent of the ASHAs, which were later transcribed. Descriptive statistics were computed for the closed-ended questions, and inductive thematic analysis was adopted for the open-ended questions [14] 


\section{POSITIONALITY}

We have been working in the maternal and child health field with a dedicated focus on understanding and improving the working conditions of ASHAs since 2016. We are all native language speakers as the ASHAs and also belong to the same socio-cultural geographical regions. Three authors identify themselves as female, one a young mother herself, and the fourth is a senior male academician. By being born and brought up in the North-Indian contexts, including staying in rural regions, we have been acknowledging the hardships women face in our culture. We strive to develop a better sense of how lay and poor village women, when they become healthworkers, negotiate their domestic and official responsibilities while being on intersections and often engage with feminist theory lenses in our approach.

We conducted our research with an open mindset grounding it in the raw data.

\section{FINDINGS}

In this section, we report 1.) the analysis of the questions asked by the ASHAs by discovering the topics and the kinds of gaps they indicate in the knowledge and skills, 2.) analysis of the reference material given to ASHAs and its mapping backward with the questions to understand the coverage, and 3.) analysis of the ASHAs perceptions and experiences on our training intervention and existing face to face training methods.

\subsection{Question Analysis}

We analyzed a total of 1178 questions of ASHAs. Each question was analyzed to find the topic and sub-topic it contained and the type of knowledge-seeking. Table 2 shows the 15 topics and their frequencies on which ASHAs asked their questions. Taking together, all these 15 topics cut across the spectrum of reproductive, maternal, newborn, child, and adolescent health, with four major clusters centered on antenatal care, health and happiness of mother (postdelivery), breastfeeding, and newborn care. Since discussing all of them is out of scope for this paper, we selected the first eight topics covering the six training topics (first six) and the top four topics with the highest frequency.

ASHAs' questions are concerned largely with field cases. Through these, ASHAs sought information and advice on the various kinds of health problems their clients faced. Our first measure of analysis identified the topics and sub-topics of the problems to develop a sense of the prevalent areas on which ASHAs need knowledge support. The second measure of analysis identified the level of knowledge and skill gaps ASHAs showed while discussing these field cases. To do this, we referred to the official training material of ASHAs that defines an ASHA's role in dealing with health problems based on three levels. For mild health problems, an ASHA should be able to provide guidance to families, including giving basic treatments. For moderate problems, which are currently non-severe but need attention, an ASHA should be able to guide families by watching for early signs of danger and making referrals if necessary. Moreover, for severe problems, an ASHA should facilitate early detection and making referrals.
Table 2: Question Topics and Frequency

\begin{tabular}{|c|c|}
\hline Topic & Count $(\%)$ \\
\hline Breastfeeding* & $239(20.29 \%)$ \\
\hline Danger Signs in Mother* & $29(2.46 \%)$ \\
\hline Danger signs in Newborn* & $78(6.62 \%)$ \\
\hline Health and Happiness in Mother* & $123(10.44 \%)$ \\
\hline Newborn Temperature Control $^{*}$ & $36(3.06 \%)$ \\
\hline Play and Communication with Newborn* & $13(1.10 \%)$ \\
\hline Antenatal Care ${ }^{*}$ & $214(18.17 \%)$ \\
\hline Newborn Care* & $265(22.50 \%)$ \\
\hline Gynaecological Issues & $93(7.89 \%)$ \\
\hline Adolescent & $10(0.85 \%)$ \\
\hline Older child (>2 years) & $19(1.16 \%)$ \\
\hline Older child (1-6 months) & $13(1.10 \%)$ \\
\hline Older child (6 months-2 years) & $25(2.12 \%)$ \\
\hline Top feed & $7(0.17 \%)$ \\
\hline Miscellaneous & $2(0.17 \%)$ \\
\hline total & 1178 \\
\hline
\end{tabular}

* topics selected for the analysis

\subsection{Knowledge Seeking Type}

Given a question, we gauged the severity of the problem mentioned in it by understanding its response given by the doctor and validating that from the reference material. Doing this for all questions, we observed three forms of knowledge-seeking as follows:

- Basic Knowledge Improvement: represented information seeking that showed gaps in the essential knowledge and skills to execute the defined roles of ASHAs across all the three levels of problems - mild, moderate, and serious. For instance, in mild problems, ASHAs lacked understanding of the problems, causes, and the simple solutions to be provided to the families; in moderate problems, ASHAs lacked understanding of symptoms and the scope of help they can provide to families; and in severe problems, ASHAs showed lack of knowledge in referring families to the right facility and in providing guidance till families receive care or after receiving the care (see table 3 for examples). The questions were mainly of 'what' and 'how' forms, seeking factual and procedural knowledge for problem-solving [105]. In our data, we found 892 questions (75\%) showing information seeking basic knowledge improvement. From the total 337 ASHAs who asked questions, 323 asked on basic knowledge improvement.

- Knowledge Enhancement - In this form of information seeking, ASHAs showed interest in knowing more than the factual knowledge by focusing more on the reasoning, causes, and concepts. The problems ranged from mild to moderate (see examples in table 3). Typically the questions under this category had "why" form of seeking, e.g. "why do healthy infants get milk in ears", "why do newborns catch jaundice on the third day after birth", "what is the reason of menstruation not getting stopped during pregnancy for some women". Out of 
Table 3: Question Categorization by Severity Level and Type of Information Seeking

\begin{tabular}{|c|c|}
\hline Question Classification & Rationale \\
\hline $\begin{array}{l}\text { MILD } \\
\text { "Post-delivery mothers usually develop constipation, } \\
\text { what advice should we give?" (Basic Knowledge) }\end{array}$ & $\begin{array}{l}\text { - Constipation is a very common problem most mothers } \\
\text { face after delivery. It usually takes few days for normal } \\
\text { bowel movements to occur [90]. } \\
\text { - An ASHA can help mothers by providing advice on } \\
\text { water consumption, food intake and physical exercises }\end{array}$ \\
\hline $\begin{array}{l}\text { "Many newborns develop jaundice on birth, } \\
\text { what is the reason for that?"(Knowledge Enhancement) }\end{array}$ & $\begin{array}{l}\text { - Newborn jaundice is a common usually harmless condition, } \\
\text { particularly in babies born before } 38 \text { weeks' gestation (preterm babies) } \\
\text { and some breast-fed babies. Mostly mild, it starts } 2-3 \text { days after birth } \\
\text { and without treatment tend to get better after } 2 \text { weeks [ } 75,92] \text {. } \\
\text { - ASHAs should know how to check yellowish discoloration } \\
\text { of skin and eyes correctly and advice mothers to continue breastfeeding. }\end{array}$ \\
\hline $\begin{array}{l}\text { MODERATE } \\
\text { "During HBPNC visits, if we observe small pimples } \\
\text { on the body of newborn then what should we do?" } \\
\text { (Basic Knowledge) }\end{array}$ & $\begin{array}{l}\text {-Skin conditions in newborn can range from mild diaper rash } \\
\text { to milia to a more severe pustules and blisters [75]. } \\
\text { - ASHAs should be able to identify the severity as more than } \\
10 \text { pustules are dangerous and need referral. }\end{array}$ \\
\hline $\begin{array}{l}\text { "If a pregnant lady in her third month experiences bleeding, } \\
\text { but ultrasound report comes fine, will everything be alright later on? " } \\
\text { (Knowledge Enhancement) }\end{array}$ & $\begin{array}{l}\text {-Vaginal bleeding is a common first trimester complication, } \\
\text { often considered to be a sign of a problem in pregnancy. } \\
\text { Bleeding multiple times do have more risk of preterm labour [110], } \\
\text { - ASHAs needs to be more vigilant with these mothers } \\
\text { and refer immediately if the mother reports } \\
\text { spotting or bleeding at any point in pregnancy. }\end{array}$ \\
\hline $\begin{array}{l}\text { SEVERE } \\
\text { "What should we recommend in the case of reduced } \\
\text { amniotic fluid in six-month pregnancy stage?"(Advanced Case) }\end{array}$ & $\begin{array}{l}\text { - Oligohydramnios occurs in about } 1-5 \% \text { of pregnancies at term. } \\
\text { oligohydramnios is associated with a high rate of pregnancy } \\
\text { complications and increased perinatal morbidity and mortality }[10,62] \\
\text { - Such cases need expert advice }\end{array}$ \\
\hline $\begin{array}{l}\text { "Each time the child breathes in, the chest pulls in, the skin } \\
\text { on the neck pulls in, the child is also not breastfeeding. } \\
\text { In such as case, what advice should we give and where should } \\
\text { we refer?" (Basic Knowledge) }\end{array}$ & $\begin{array}{l}\text { - Chest indrawing in a child with cough or difficult breathing indicates } \\
\text { that the child has pneumonia, should be referred to the hospital [75]. } \\
\text { - Aiding in management of acute respiratory infection is defined } \\
\text { as one of the key task under the skills for } \\
\text { recognizing danger signs of children. ASHAs are supposed to have good } \\
\text { knowledge on recognizing and classifying breathing patterns }\end{array}$ \\
\hline
\end{tabular}

337, 94 ASHAs showed interest in knowledge enhancement and asked 147 questions (13\%).

- Advanced Cases - The questions under this category represented health conditions which were stated advanced for ASHAs such that either the health problems, as already diagnosed, are complex or in severe condition. ASHAs sought general knowledge on the problems. For such cases, the doctor recommended ASHAs to not focus on understanding the problem but on providing support to the families (see examples in the table 3). The training materials of the ASHAs also did not cover such cases. The total number of questions under this category was 139 (12\%).

We will now illustrate the gaps in the essential basic knowledge and skills in the next section, which is our major finding. As mentioned previously, we discuss these through the eight selected topics, namely breastfeeding, danger signs in mother, danger signs in newborn, health and happiness in mothers, play and communication with newborn, newborn temperature control, antenatal care, and newborn care, which composed of 997 questions.

\subsection{Illustration of Knowledge Gaps}

6.3.1 Routine Patient Care. By making home visits, ASHAs get first-hand accounts of their fellow community members' routine health experiences. This reflection held a major theme in our results. $60 \%$ of the questions represented day to daycare concerns and common health problems (see table 4 and figure 1). The maximum number of queries on antenatal care (87 of 214) were on common problems and discomfort due to pregnancy changes, e.g., cramps, heartburn, varicose veins, etc. The recommended remedies to these concerns are related to self-care and lifestyle changes. In addition to developing adequate factual knowledge on guiding these, as observed from the doctor's answers, the key aspect was to tailor the information according to individual needs and address the prevalent perceived lack of willingness towards health adherence in an effective manner. For instance, even though Anemia is one of the biggest threat to maternal health in rural India and given the focus on increasing adherence to the consumption of iron and folic acid supplements in the antenatal care in the training material, ASHAs lacked basic knowledge about the intake and side effects of the supplements "Should iron tablets be taken during day time or after dinner?", "Can iron tablet cause itching?".

The common questions post-delivery constituted of problems related to delivery discomforts and body changes (42 questions, see the graph - health and happiness of mother in the figure 1), e.g., bleeding, discharge, stitches pain, etc. The second most sought topic in this phase was contraception (23), mainly concerning the Copper-T method (13). Few questions sought further knowledge on the safety extent of breastfeeding as a natural contraceptive.

Questions on the diet highlighted the prevalence of deep-rooted traditional practices (18). Corroborating with the various kinds of 
dietary restrictions during pregnancy and post-childbirth as reported by the prior studies [108]. ASHAs sought both reassurance and clarification of these practices. In only one question, an ASHA acknowledged the practices to be harmful and sought counseling tips. TThe rest questions showed how ASHAs carried on the misbeliefs and lacked scientific knowledge, for example, "How many days after delivery should a mother take a bath?", "Do infants frequently pass stools because mothers consume ghee (clarified butter)? Should less ghee be given to such a mother or not given altogether?", "After the delivery if a mother is not given enough food and is given hot water so that her belly does not protrude out. Is this okay?" Queries on newborn care sought knowledge on the daily care of newborns (102), common health problems (43), and weight tracking (36). Examples of sub-topics under the routine care topic were umbilical stump care, massaging technique, bathing frequency, eye care, sleep routine, etc. Some of these, such as stump care, bathing frequency, and eye care, showed the need to address the communities' prevalent malpractices. Monitoring the growth of newborns means tracking newborn weight gain week by week for the first 42 days. This is one of the critical knowledge components concerning newborn survival. However, the ASHAs showed significant gaps. In 32 out of 36 questions, ASHAs showed a complete lack of knowledge on the appropriate weight gain ranges, e.g. "How much weight a newborn should gain in a week?". Also, ASHAs did not seem to have a good understanding of the factors associated with newborn growth, as shown below:

ASHA13, Group11: "If a newborn's weight does not increase even if he/she is breastfed well then what advice should we give?"

Doctor: "If this is happening, then the mother is not feeding the baby properly. You or the mother might feel that the baby is being fed properly when he/she is not. Proper feeding means that any worry does not bind the mother. It means that she is feeding the baby multiple times, both in the day and night. It means that she feeds only from one side at a time. The baby should not just suck on the nipple but put pressure on the areola, the baby's chin must be touching the mother's chest".

The topic of newborn temperature control, which requires more hands-on skills, reflected a poor understanding of the topic by ASHAs. 22 out of 36 questions were on body temperature. ASHAs showed confusion on measuring temperature, interpreting temperature readings, and taking corrective action, e.g., "What should be the newborn body temperature in summers and winters?", "If on touching a child feels warm but otherwise his/her temperature is normal then what we should do?" The topic of play and communication had very few questions (13) covering basic questions on newborn development, five senses, and mother-child play and bond, all of the mild severity.

6.3.2 Recognition of Danger signs. The second most important information seeking represented problems of moderate to severe nature, holding $40 \%$ of queries (see table 4 and figure 2,3). These were mainly about illnesses and diseases where ASHAs sought to improve their knowledge on distinguishing between danger and normal signs and necessary actions to take (a majority of the
Table 4: Questions Distribution According to Severity

\begin{tabular}{lccc}
\hline Topic & Mild & Moderate & Severe \\
\hline Danger Signs in Mother & 0 & 8 & 21 \\
Danger signs in Newborn & 0 & 25 & 53 \\
Health and Happiness in Mother & 92 & 24 & 0 \\
Newborn Temperature Control & 26 & 10 & 0 \\
Play and Communication with & 12 & 1 & 0 \\
Newborn & & & \\
Antenatal & 87 & 71 & 56 \\
Newborn Care & 231 & 33 & 1 \\
\hline Total & $448(60 \%)$ & $172(23 \%)$ & $131(17 \%)$ \\
\hline
\end{tabular}

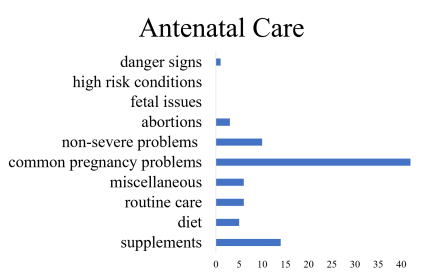

Health and Happiness of Mother
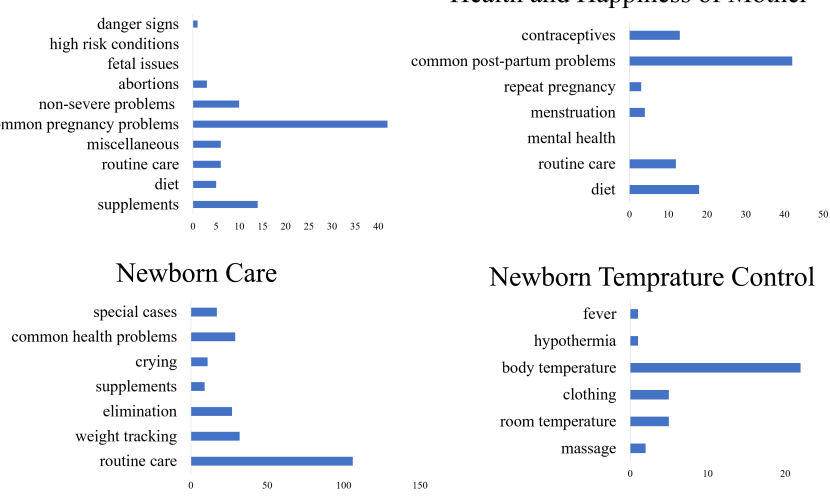

Play and Communication

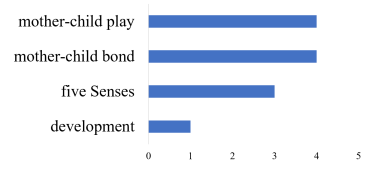

Newborn Temprature Control

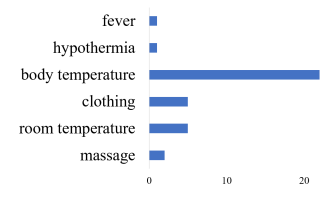

(n)

Figure 1: Topics and sub-topics of questions containing problems of mild severity

questions under danger signs represented severe conditions - shown in figure 3). The questions showed gaps in basic knowledge, for example, post-partum hemorrhage is one of the most common life-threatening danger sign, had maximum questions in danger signs in the mother category (11 questions, see figure 3 ), that is also covered in the training curriculum. However, ASHAs did not show clear understanding on recognizing the severity of bleeding such as "If after the delivery a mother changes her pad 8-10 times in a day, then is it a danger sign?", "After the delivery if the mother experiences heavy bleeding then what should be done?" Likewise, 33 of 78 questions under danger signs in newborns reflected gaps in basic knowledge and skills (25 moderate and 53 severe). The prominent areas of questions were newborn diseases and congenital disabilities, followed by growth, breathing complications, and skin problems.

6.3.3 Breastfeeding Support. Breastfeeding, as a topic, emerged as an independent theme. Since breastfeeding is not a health problem but a natural phenomenon that requires skilled support, the 


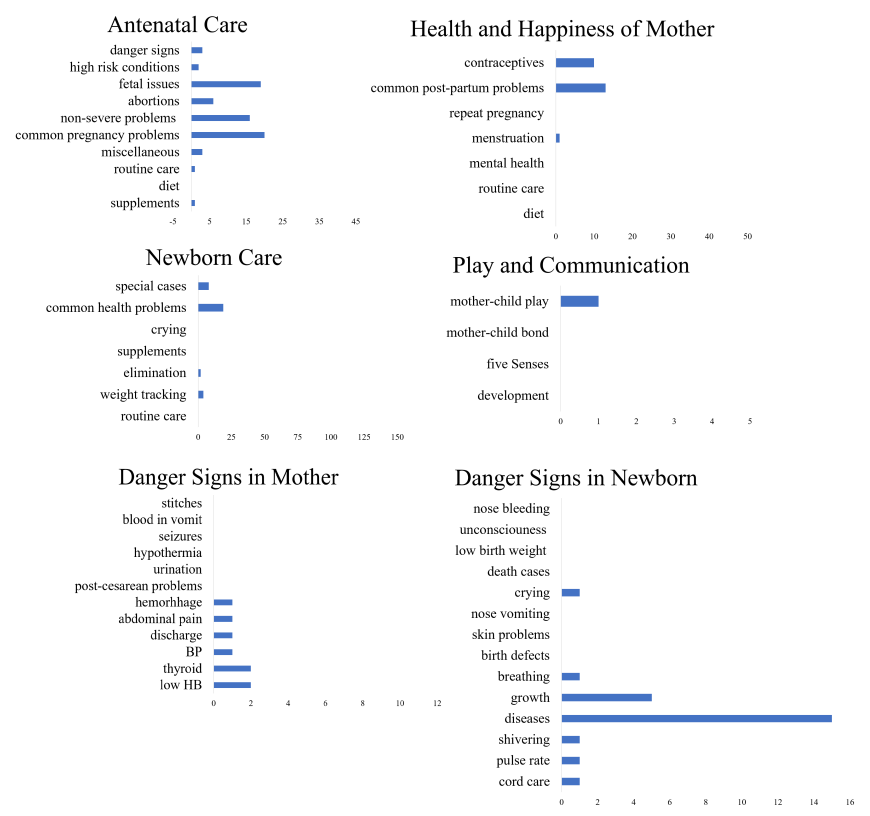

Figure 2: Topics and sub-topics of questions containing problems of moderate severity
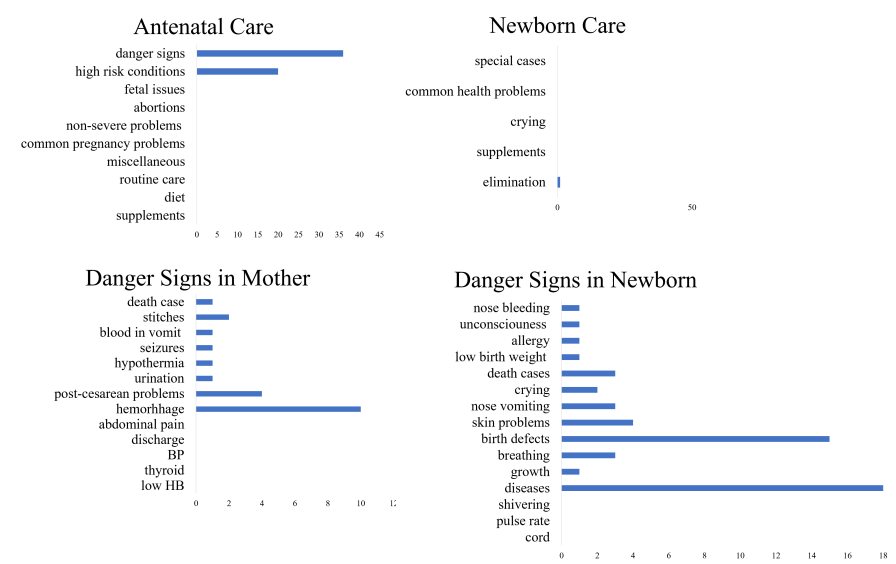

Figure 3: Topics and sub-topics of questions containing problems of severe severity

questions were not judged as mild, moderate, or serious. Providing breastfeeding support is a core skill for ASHA and forms a part of fundamental knowledge improvement. We categorized the questions further into six sub-topics, as shown in the table 5.

Queries on the early initiation of breastfeeding (37) covered seeking tips on encouraging new mothers, providing counseling on initial concerns of milk supply, and teaching breastfeeding techniques. A key concern raised here was about how to ensure initiation of breastfeeding within one hour of delivery in case of cesarean sections, "When a child is delivered via cesarean, then mother remains unconscious for hours, in such a situation how can we facilitate the initiation of breastfeeding with the help of doctors?" Cesarean section delivery adversely impact breastfeeding initiation is a growing public health concern [89]. It has been reported that many birth facilities do not follow the recommended best practices, e.g., keeping mothers and newborns together, facilitating skin-to-skin contact etc. Here, ASHAs also sought to understand how they could better support breastfeeding in existing hospital environments.

The core gap in the knowledge of ASHAs emerged about breastfeeding physiology and technique. The questions and explanations covered under the topics - breastfeeding technique (39 questions), breast problems (43 questions), and insufficient milk supply (36), and child refusing breastmilk (13) in perception-based problems, majorly showed this. The root cause highlighted by the doctor for all these associated with ineffective breastfeeding technique - poor positioning, inadequate latching, and inadequate feeding frequency. A sample conversation is given below:

ASHA5, Group4: "If the mother is breastfeeding the baby yet a lump forms in the breast, what should she do about it?"

Doctor: "Lump formation even after breastfeeding is a sign that mother's breast is not getting emptied. That is why she must not let her/him suckle the nipple when the mother feeds the baby. The baby should suckle on the areola, and then milk will come in nicely. if a lump has formed and is not dissolving, then you should not take any risk and refer the mother."

Maternal perceptions of breastmilk insufficiency is a common barrier to exclusive breastfeeding [40]. However, ASHAs' mentions of the associated problems seemed to be no different from mothers. ASHAs even carried the prevalent community misbeliefs, for example, considering breastmilk as being harmful to children. The understanding of exclusive breastfeeding was also observed as limited. ASHAs showed confusion and presented a variety of situations to understand whether to continue breastfeeding or not. For example, "If a newborn has red rash/bumps at birth, can mother breastfeed the baby?", "A mother has a six-month-old baby and now she is again pregnant, can she breastfeed her six-month-old baby?".

To know the current training duration on breastfeeding, we investigated two training officials of the region selected in our study. The breastfeeding topic is covered in less than half a day as part of the Home-Based Post-Natal Care training module. Besides this, there is no other specific training.

\subsection{Mapping of Questions to Reference Material}

The reading reference material of ASHAs is spread across seven modules, corresponds to their training modules. The official training of ASHAs covers these modules in two phases. The first phase covers module 1 to module 5 , also called the induction training program, and the second phase covers module 6 and module 7 to impart skills on Home Based Post Natal Care, which we chose as our training topic. Overall, the seven modules cover 19 themes, which are covered in 23 days of training. These themes are further organized into lessons such that each lesson is 2-3 pages long and can be completed in half an hour, as mentioned in the books. Every lesson covers both knowledge and some skills aspects presented in 
Table 5: Breastfeeding Queries Typology and Definition

\begin{tabular}{|c|c|c|c|}
\hline Category (count) & Sub-Category (count) & Category (count) & Sub-Category (count) \\
\hline $\begin{array}{l}\text { Early Initiation of } \\
\text { Breastfeeding (37) }\end{array}$ & $\begin{array}{l}\text { cesarean deliveries } \\
\text { milk production \& } \\
\text { newborn suckling concerns }\end{array}$ & $\begin{array}{l}\text { Perception-based (73) } \\
\text { problems }\end{array}$ & $\begin{array}{l}\text { insufficient milk supply (36) } \\
\text { child refusing breastmilk (13) } \\
\text { breastmilk is harmful (14) } \\
\text { mother not willing (4) }\end{array}$ \\
\hline $\begin{array}{l}\text { Breastfeeding } \\
\text { Technique } \\
(39)\end{array}$ & $\begin{array}{l}\text { Positioning (4) } \\
\text { attachment (3) } \\
\text { frequency ( } 4 \text { ) } \\
\text { one sided nursing (4) } \\
\text { twins ( } 6) \\
\text { triplets (1) } \\
\text { low birth weight babies (11) } \\
\text { inactive babies (1) }\end{array}$ & $\begin{array}{l}\text { Breastfeeding } \\
\text { discontinuation (38) }\end{array}$ & $\begin{array}{l}\text { mother is sick (31) } \\
\text { child is sick (2) } \\
\text { mother is pregnant (5) }\end{array}$ \\
\hline Breast Problems (43) & $\begin{array}{l}\text { engorgement ( } 3) \\
\text { cracked nipple }(14) \\
\text { lump in breast }(10) \\
\text { nipple shape }(14) \\
\text { breast size }(2)\end{array}$ & Miscellaneous (9) & $\begin{array}{l}\text { myth }(1) \\
\text { introduction of water }(2) \\
\text { diet (1) } \\
\text { newborn death (2) } \\
\text { dos and don'ts (2) }\end{array}$ \\
\hline
\end{tabular}

simple language with fair use of illustrations and highlights. The information is presented in the form of a bulleted list that can be read and skimmed. However, the books do not provide information in the format of frequently asked questions, and one needs to search for information topic wise.

6.4.1 Questions Coverage Proportion. We could classify the coverage of ASHA questions into three categories. First, the questions for which answers could be directly found in the content of the associated topic. Second, questions for which answers could be derived only partially because the present information is not complete enough to fully understand the concept or find a solution to a problem (a sample depicted in the table 6 ). Third, the questions whose answers could not be found either because the associated topic is not covered or the underlying problem is too specific. Of 997 questions, only $22 \%$ were covered, $15 \%$ were partially covered, and $63 \%$ were not covered.

\section{Table 6: Sample of a partially covered question}

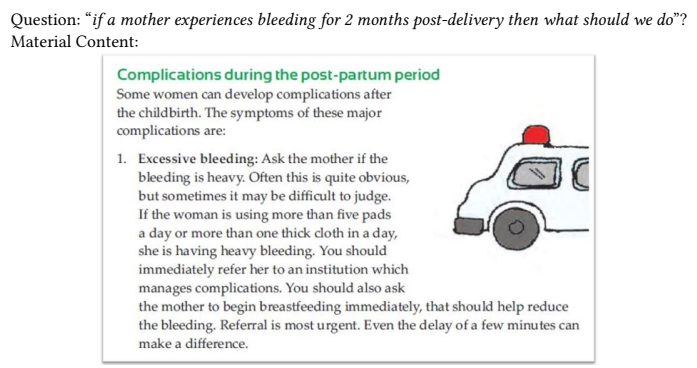

Looking more into what is not covered, we now present our findings across the three themes discussed in the previous section routine patient care, recognition of danger signs, and breastfeeding. Mapping a coarse analysis for the topics - antenatal, health and happiness of mother, newborn care, newborn temperature control, and play and communication with more than $70 \%$ of the problems as mild, $69 \%$ were found to be not covered in the reference material.

Table 7: Topic Wise Coverage

\begin{tabular}{llll|l}
\hline Topic & Covered & Not Covered & Partially Covered & Total \\
\hline Breastfeeding & 85 & 99 & 55 & 239 \\
Danger sign in mother & 8 & 12 & 9 & 29 \\
Danger Signs in Newborn & 10 & 64 & 4 & 78 \\
Newborn Care & 48 & 188 & 29 & 265 \\
Newborn Temperature Control & 11 & 11 & 14 & 36 \\
Play and Communication 0 & 12 & 1 & 13 & \\
Health and Happiness in Mother & 10 & 104 & 9 & 123 \\
Antenatal & 45 & 136 & 33 & 214 \\
\hline & & & & \\
Total & $217(22 \%)$ & $626(63 \%)$ & $154(15 \%)$ & 997 \\
\hline
\end{tabular}

6.4.2 Coverage Analysis. In the antenatal care topic with 61 questions under common pregnancy discomforts, only 12 were covered (see table 7). Forty-four were not covered, and five were partially covered. From all the common problems, only discharge and swelling in the body were covered as part of content under danger signs. Rest of the problem types which most mothers face, however, were missing in the reference material. The overall content on antenatal care (covered into two chapters over 20 pages) mainly talks about educating women on making antenatal contacts, watching for early signs of dangers, caring for anemia, educating on the importance of nutritious diet and iron supplements, helping women receive food-related services from nearest centers, and developing plans for birth preparedness $[66,67]$. However, it does not provide any pointers on common discomforts, dos and don'ts, and general care information.

Likewise, from the 55 questions on common post-partum problems in the health and happiness of mother topic, only four were covered. Three of these, two on fever and one on bleeding, were covered under the section on complications during the post-partum period [68]. The fourth question about iron tablets' side effects was covered indirectly in the diet section under antenatal care. All 
other types of problems, such as pains, bleeding, vaginal discharge, stitches discomforts, skin problems, hemorrhoids, and bowel movements were not covered. The diet-related questions which sought clarifications of prevalent misconceptions (18/18), doubts and concerns related to body image and routine care (10/12), counseling support (6/7), menstruation (5/5), repeated pregnancy (3/3) were not covered. 10 out of 23 questions on contraception had missed topics on breastfeeding as a contraceptive and oral and injectable hormonal contraception [69].

In newborn temperature control (36 questions), 11 were covered, 14 were partially covered. The remaining 11 that were not covered questions were about room temperature and methods of measuring temperature. The play and communication were not covered in the material at all.

On danger signs, 12 of 29 danger signs in mother (41\%) and 64 of 78 questions on danger signs in the newborn (82\%) were not covered (see table 7). On danger signs in mother, post-cesarean problems, thyroid problems were not covered [68]. From the 11 questions on excessive bleeding, six were directly covered. The remaining 5 represented case-specific problems, e.g. "There is a case in which the bleeding of the mother stopped after 15 days of delivery, however, started again after some days then what to do?". Of these, four were partially covered, and one was not covered.

For Danger signs in newborns, the not covered topics majorly came from newborn diseases (30), skin problems (4), and congenital disabilities (15). A common disease that most newborns develop immediately after birth is jaundice, which mostly remains mild to moderate but becomes serious and needs referral and is listed as a common danger sign. There were 11 questions on jaundice, all of which were not covered. We found only one line of information on jaundice covered as part of the section - Neonatal Sepsis: Diagnosis and Management, written as "When to refer baby - becomes yellow (jaundice) on the first day or jaundice persists after 14 days" [69]. Congenital disabilities, which have been reported to high in rural India, constituted of 15 questions and were included in our training intervention were also not found to be covered.

Finally, in the 239 questions of breastfeeding, 85 (36\%) were covered, $99(41 \%)$ were not covered and $55(23 \%)$ were partially covered (see table 7). The prominent areas in which the questions were not covered included breastfeeding technique (15), discontinuation of breastfeeding due to sickness of a mother or newborn (38), and attitudinal and perception-based problems (27).

The 15 questions not covered under breastfeeding technique included specific problems, cases of twins (6), triples(1), low birth weight cases (4), and one-sided nursing (3). The content concerning the continuation of breastfeeding during mother's or child's sickness was inadequately covered. The section on "Engorged and painful breasts (very full breasts)", in Book 6, contained one statement as "She should continue to breastfeed (from both sides) even if she is taking antibiotics". Under the category attitudinal and perception-based problems, there were total of 73 questions that constituted four types of questions - not enough milk, a child refusing breastmilk, perception of breastmilk being harmful, and mother not willing to breastfeed (see table 5). From these, 32 were covered, 29 on not enough milk and 3 on child refusing to breastfeed, the rest two categories as mother's perception of breastmilk being harmful (11), which is a myth and mothers' unwillingness to feed were not addressed (3). The counseling component, which is a crucial skill for addressing perception-based problems, was not explicitly explained. There were only general tips for communicating with mothers and mentions of causes. For instance, the section on "not enough milk" only list causes such as, e.g., mother's anxiety, exhaustion, insecurity, and inadequate family support without elaboration.

\subsection{Training Experience}

In this section, we present the interview findings of the training experiences of ASHAs and their feedback for the future.

6.5.1 Perceived Benefits. While most of the ASHAs liked all the three features of the systems - content listening, Q\&A with the doctor, and peer to peer group discussion - the interaction with the doctor was mostly strongly preferred.

With a dedicated time allocated to questioning and answering with a field expert every day, the ASHAs could effectively discuss their field problems. This was reported as important because of the lack of regular forums for problem-solving.

Moreover, ASHAs acknowledged the respect they received as health workers. Prior studies have reported issues with recognition of ASHAs as health workers, which was reflected in the views, for example, "One benefit that I felt was that there is someone who understands us and would listen to us. Because sometimes our doctors and nurses are not able to understand our point. But they (trainers) listened to us very patiently and carefully." (ASHA1, Group11)

By receiving expert guidance, ASHAs felt more confident in facing their clients, expressed as follows.

"If we are unable to give them [clients] correct guidance, we feel like a failure, but when we are able to guide them effectively, then we feel successful and that we have executed our responsibility correctly. So that is what I asked the doctor about and he explained very nicely that things are like this, do this, do that, if they still do not understand, counsel them like this. Moreover, the rest of the things we learn by practice". (ASHA2, Group16)

On the extent of interaction with trainers in existing face-to-face training methods, ASHAs commented that while they interact with trainers in the sessions, that is more limited to clarifications of doubts on the training topics. Training schedule remains typically tight with limited scope for discussion on day to day field queries.

In addition to getting help in problem-solving, the training intervention served the purpose of refresher training. ASHAs said that they receive training as and when new programs are launched; however, they do not get opportunities to refresh older topics. The use of reference material did not seem to be effectively used. While mentioning the benefit of direct interaction with experts, ASHAs said that they do not get time to read the material and prefer verbal communication.

6.5.2 Usability. As reported by prior studies, sub-optimal cellular connectivity became a key challenge in our deployment also $[106,109]$. Due to lower signal strength, call drops were frequent. According to the logs captured by our systems, $86 \%$ of ASHAs $(428 / 500)$ experienced at least one call drop in every session, and 30 ASHAs experienced more than three call drops in a session (max 
was 7). Further, troubleshooting activities due to poor internet connectivity at the trainers' end and issues in the server-side added to the inconvenience. When we asked the ASHAs about their experience of attending the sessions, 20 (out of 48) mentioned the connection problems and mainly complained about the voice quality issues. "On dropping, we used to get the callbacks, but due to voice breaks, we could not hear the audios properly. This happened for the first two days when I attended the sessions downstairs, I then used to sit at the terrace." As a result, one ASHA even preferred a traditional brick and mortar training mode over this mobile-based.

Another challenge observed by some of the ASHAs related to making adjustments with the training timings. The morning hours did not suit them as they had to be in the fields for usual ASHA work. Even after prior notice, they had to skip some sessions, sometimes because of meetings with supervisors and other times to serve urgent duties. These were on top of domestic responsibilities which are typically quite demanding, expressed as "By the way, it is also important that housework is also necessary, work had to be done at that time. I used to get the phone call while cooking, and you know, family members would then speak."

6.5.3 Feedback. We asked ASHAs to share feedback on improving the system and gave them probes around system features, training topics, and training frequency. Feature-wise, ASHAs suggested making the audios available to them for offline use over WhatsApp or otherwise. Some ASHAs also wished for videos so that they can show them to their clients during home visits. Interaction featurewise, ASHAs suggested to keep all the three and did not show an inclination towards using the system with only peer-to-peer interaction feature. An expert's presence was deemed important to have authority and authenticity in the information. Concerning timings, a suggestion was to keep their departments well informed and not just immediate supervisors. For the frequency of mobilebased training, 31\% desired for once in three months, $40 \%$ desired for once in six months, and $29 \%$ once in a year.

Topic-wise, ASHAs found breastfeeding and danger signs as the two most impactful areas to learn on. ASHAs said that as they frequently face complex health situations such as recurrent abortions, pre-term deliveries, newborn deaths, they need more training to develop a good understanding of recognizing dangers early on.

\section{DISCUSSION}

A unique aspect of ASHAs is that they are embedded in the communities they serve. As fellow community residents, ASHAs get to interact with their clients on a routine basis. This places ASHAs in a strategic position to receive first-hand accounts of the health experiences of families. As we observed, most of the questions pertained to day to day care concerns and common health problems (more than $60 \%$ ). This is the area that requires the most improvement ASHAs showed significant gaps in their basic knowledge to address their clients' needs. We now discuss three potential areas to focus on.

\subsection{Improvement in Reference Material}

We observed two concerns with the use of existing reference material. First, the material is not used by ASHAs, and second, the material does not sufficiently cover common queries.
Regarding the non-use of material, though we did a coarse level of inquiry, we noted that ASHAs prefer discussion-based learning over reading text. ASHAs find reading text to be time-consuming and want something that can directly answer their questions. This is one of the reasons why ASHAs favored the interaction with the doctor as the most useful feature of our system. While the requirement of enhancing the content design in the form of FAQs and problem-solving exercises is clear, it is essential to investigate the usability further. Understanding how ASHAs comprehend the material will help recognize better the cognition challenges ASHAs might have due to considerable variation in their education and literacy levels [53].

Likewise, the investigation should also extend to other forms, such as those designed specifically for use in the fields. Prior studies have reported less use of pictorial flip charts by ASHAs in their home visits.

Project - Mobile Kunji - provides a successful example of using flashcards in this case [2]. The project combines flashcards with an IVR application to assist ASHAs in their dialogue with women in-home visits. Flashcards are an effective way of reinforcing spaced learning. Flashcards are a handy resource that has been found useful for every stage of class [43]. Flashcards are also more appealing to visual learners like ASHAs who generally have low exposure to formal schooling. Further usage of Flashcards or alternate interventions may be explored to support ASHAs in the field.

The second aspect is to improve the coverage of the existing reference material. Overall, we found $63 \%$ of the questions to be not covered - 69\% in routine patient care, $70 \%$ in danger signs, and $41 \%$ in breastfeeding were not covered. By developing a typology of the questions, we provide the coverage information further in detail and create a resource for future work. This calls for enhancing the existing material and thinking of other solutions to cater to the information needs of ASHAs. Further, we also suggest to improve the content analysis by going deeper into understanding the nature of questions - fact-based or case-based etc.

\subsection{Design Guidelines for Mobile-Based Training Solutions}

Our findings showed the importance of providing a forum for interaction with experts to ASHAs. In addition to knowledge improvement, two key benefits highlighted were improved communication with clients and problem-solving skills. ASHAs tried to learn by discussing their field cases with the expert. This helped them understand the systematic ways of making conversations with their clients and arrive at appropriate decision-making. The majority of the ASHAs desired such mobile-based training at a frequency of twice in a year. This is encouraging to find. However, given that there are acute shortages of experts in the developing settings [77], it is crucial to employ better training designs. So that technology use and human effort can be balanced. This suggests shifting the effort of answering repeated and fact-based queries to the machine and use experts' resources for improving decision-making skills. This also implies incorporating automation in the training process with a focus on the use of pedagogical techniques to encourage self-, and peer-learning [102, 104, 106, 108]. Prior work has shown promising results of employing spaced education, and 
offline learning formats [33]. Designing coursewares for CHWs that can be delivered through inexpensive feature phones is a potential research direction.

Further, given that the smartphone penetration is rapidly increasing in poor regions and increasing news of equipping ASHAs with smartphones $[71,80]$, it is worth to design richer applications. Our study observed that though the IVR-based platform provided an advantage of reachability, it was limited in user experience, e.g., dependency on voice-based communication. Smartphone applications will help in supporting visual learning and delivering learning resources for offline use.

Another factor to consider in providing mobile-based training interventions is the workload of ASHAs, which is not limited to just the official duties but domestic responsibilities [35]. Corroborating with the prior studies $[28,106]$, we observed time challenges with ASHAs. Due to high engagement in the fields, urgent community needs, and family responsibilities, it became difficult for some of the ASHAs to attend all the sessions. This calls for attention at multiple levels - human-centered design in HCI, program management, and policymaking. Designs taking care of the intersections CHWs are positioned at have the potential to further the aspirations of CHWs in becoming a credible health workforce [35].

\subsection{Directed Attention on Breastfeeding}

There are two critical reasons to highlight knowledge building around breastfeeding. First, from the public health domain, there are increasing calls for taking action on promoting breastfeeding $[50,76]$. Despite the recognition of innumerable benefits of breastfeeding for the mother and infant's health [38], the rates of breastfeeding are low worldwide and among all strata of populations $[32,50]$. Second, given that there is a high link between infant mortality and poor breastfeeding, it is more important to improve community health workers' capacity to support breastfeeding. The Lancet series on child survival identified breastfeeding interventions to have the potential to prevent $13 \%$ of all under- 5 deaths in developing areas of the world, ranking it as the most important preventive measure [38].

In our intervention, we observed that ASHAs lacked knowledge of breastfeeding physiology and technique and skills to counsel women and address misperceptions and prevailing misbeliefs on milk supply.

The WHO/UNICEF Breastfeeding hospital Initiative provides a detailed guideline "Ten Steps to Successful Breastfeeding" on supporting breastfeeding for facilities providing maternity and newborn [79]. It recommends that all health care staff who have any form of contact with mothers, infants and/or children must receive at least 18 hours (five days) of training on breastfeeding and lactation management in total, with a minimum of 3 hours of supervised clinical experience and cover at least eight steps. Whereas, as we found, ASHAs nearly remain untrained by receiving only 2-4 hours of overview on breastfeeding as part of their HBPNC training. Although ASHAs are viewed as an effective source for providing breastfeeding counseling as they are women who themselves have breastfed babies and have the potential to play the role of "experience-based experts" [17], there are serious gaps in skilling them. This raises questions on the policymakers, program managers, and the National Health Mission, as to how they could aim to provide effective post-natal care without emphasis on the training of breastfeeding for ASHAs. The current support system to skill ASHAs on breastfeeding is inadequate and requires specific design innovations to address the challenge.

\section{CONCLUSION}

Our study is the first of its kind in terms of scale and focuses on understanding the learning and training needs of CHWs. Our findings of the inadequate content and its presentation, more handson support for handling in-the-field queries, and a strong emphasis on developing skills for promoting breastfeeding complement the existing research towards providing technical support to CHWs. Our study should serve as a call to provide more support to CHWs who are otherwise left with inadequate training, insufficient care, and not enough support for their fieldwork.

\section{ACKNOWLEDGMENTS}

The research is supported by Bill and Melinda Gates Foundation award (OPP1190817). The authors would like to express special thanks of gratitude to Dr. Vijay of SWACH team and Anushka Bhandari.

\section{REFERENCES}

[1] BBC Media Action. 2020. Kilkari. https://www.rethink1000days.org/programmeoutputs/kilkari/. [Online; accessed 20-Oct-2020].

[2] BBC Media Action. 2020. Mobile Kunji. https://www.rethink1000days.org/ programme-outputs/mobile-kunji/. [Online; accessed 11-Jan-2021].

[3] Atik Ahsan and Ananya Raihan. 2013. Understanding mHealth impact among Aponjon (MAMA Bangladesh) subscribers through a phone survey in Bangladesh. In Proceedings of the Sixth International Conference on Information and Communications Technologies and Development: Notes-Volume 2. 1-4.

[4] Teresa Almeida, Rob Comber, and Madeline Balaam. 2016. HCI and Intimate Care as an Agenda for Change in Women's Health. In Proceedings of the 2016 CHI Conference on Human Factors in Computing Systems. 2599-2611.

[5] Teresa Almeida, Rob Comber, Gavin Wood, Dean Saraf, and Madeline Balaam. 2016. On looking at the vagina through Labella. In Proceedings of the $2016 \mathrm{CHI}$ Conference on Human Factors in Computing Systems. 1810-1821.

[6] Morgan G Ames. 2014. 10 Translating Magic: The Charisma of One Laptop per Child's XO Laptop in Paraguay. Beyond imported magic: Essays on science, technology, and society in Latin America (2014), 207.

[7] Nazanin Andalibi and Andrea Forte. 2018. Announcing pregnancy loss on Facebook: A decision-making framework for stigmatized disclosures on identified social network sites. In Proceedings of the 2018 CHI Conference on Human Factors in Computing Systems. 1-14.

[8] Richard Anderson, Chad Robertson, Esha Nabi, Urvashi Sahni, and Tanuja Setia. 2012. Facilitated video instruction in low resource schools. In Proceedings of the Fifth International Conference on Information and Communication Technologies and Development. 2-12.

[9] ARMMAN. 2020. mMitra. https://armman.org/mmitra/. [Online; accessed 17-Dec-2020].

[10] Asavari Ashok Bachhav and Manjushri Waikar. 2014. Low amniotic fluid index at term as a predictor of adverse perinatal outcome. The fournal of Obstetrics and Gynecology of India 64, 2 (2014), 120-123.

[11] Savita Bailur, Renee Kuriyan, Joyojeet Pal, Aishwarya Ratan, Janaki Srinivasan, Kentaro Toyama, Rajesh Veeraraghavan, Akshaya Namma Dhwani, Azim Premji, and One Roof. 2007. Review of research on rural PC kiosks. Microsoft Research India (2007).

[12] Madeline Balaam, Rob Comber, Ed Jenkins, Selina Sutton, and Andrew Garbett. 2015. FeedFinder: a location-mapping mobile application for breastfeeding women. In Proceedings of the 33rd Annual ACM Conference on Human Factors in Computing Systems. ACM, 1709-1718.

[13] Peter Barron, Joanne Peter, Amnesty E LeFevre, Jane Sebidi, Marcha Bekker, Robert Allen, Annie Neo Parsons, Peter Benjamin, and Yogan Pillay. 2018. Mobile health messaging service and helpdesk for South African mothers (MomConnect): history, successes and challenges. BMJ global health 3, Suppl 2 (2018), e000559. 
[14] Virginia Braun and Victoria Clarke. 2006. Using thematic analysis in psychology Qualitative research in psychology 3, 2 (2006), 77-101.

[15] Nadia Campo Woytuk, Marie Louise Juul Søndergaard, Marianela Ciolfi Felice, and Madeline Balaam. 2020. Touching and Being in Touch with the Menstruating Body. In Proceedings of the 2020 CHI Conference on Human Factors in Computing Systems. 1-14

[16] CHW Central. 2011. One Million Community Health Workers Technical Task Force Report. https://chwcentral.org/resources/one-million-community-healthworkers-technical-task-force-report/. [Online; accessed 22-Dec-2020].

[17] Harry M Collins and Robert Evans. 2002. The third wave of science studies: Studies of expertise and experience. Social studies of science 32, 2 (2002), 235-296.

[18] Julian Cristia, Pablo Ibarrarán, Santiago Cueto, Ana Santiago, and Eugenio Severín. 2017. Technology and child development: Evidence from the one laptop per child program. American Economic Journal: Applied Economics 9, 3 (2017), 295-320.

[19] Andrew Cross, Nakull Gupta, Brandon Liu, Vineet Nair, Abhishek Kumar, Reena Kuttan, Priyanka Ivatury, Amy Chen, Kshama Lakshman, Rashmi Rodrigues, et al. 2019. 99DOTS: a low-cost approach to monitoring and improving medication adherence. In Proceedings of the Tenth International Conference on Information and Communication Technologies and Development. 1-12.

[20] Edward Cutrell, Jacki O’Neill, Srinath Bala, B Nitish, Andrew Cross, Nakull Gupta, Viraj Kumar, and William Thies. 2015. Blended learning in Indian colleges with massively empowered classroom. In Proceedings of the Second (2015) ACM Conference on Learning@ Scale. 47-56.

[21] Nicola Dell, Jessica Crawford, Nathan Breit, Timóteo Chaluco, Aida Coelho Joseph McCord, and Gaetano Borriello. 2013. Integrating ODK Scan into the community health worker supply chain in Mozambique. In Proceedings of the Sixth International Conference on Information and Communication Technologies and Development: Full Papers-Volume 1. 228-237.

[22] Nicola Dell and Neha Kumar. 2016. The ins and outs of HCI for development In Proceedings of the 2016 CHI conference on human factors in computing systems. 2220-2232.

[23] Brian DeRenzi, Gaetano Borriello, Jonathan Jackson, Vikram S Kumar, Tapan S Parikh, Pushwaz Virk, and Neal Lesh. 2011. Mobile phone tools for field-based health care workers in low-income countries. Mount Sinai fournal of Medicine A fournal of Translational and Personalized Medicine 78, 3 (2011), 406-418.

[24] Brian DeRenzi, Leah Findlater, Jonathan Payne, Benjamin Birnbaum, Joachim Mangilima, Tapan Parikh, Gaetano Borriello, and Neal Lesh. 2012. Improving community health worker performance through automated SMS. In Proceedings of the Fifth International Conference on Information and Communication Technologies and Development. ACM, 25-34.

[25] Brian DeRenzi, Jeremy Wacksman, Nicola Dell, Scott Lee, Neal Lesh, Gaetano Borriello, and Andrew Ellner. 2016. Closing the feedback Loop: A 12-month evaluation of ASTA, a self-tracking application for ASHAs. In Proceedings of the Eighth International Conference on Information and Communication Technologies and Development. ACM, 22.

[26] Adam Fourney, Ryen W White, and Eric Horvitz. 2015. Exploring timedependent concerns about pregnancy and childbirth from search logs. In Proceedings of the 33rd Annual ACM Conference on Human Factors in Computing Systems. 737-746.

[27] International Monetary Fund. 2020. Mobile Academy. https://armman.org/ mobile-academy/. [Online; accessed 22-Dec-2020].

[28] Saji Saraswathy Gopalan, Satyanarayan Mohanty, and Ashis Das. 2012. As sessing community health workers' performance motivation: a mixed-methods approach on India's Accredited Social Health Activists (ASHA) programme. BMJ open 2, 5 (2012), e001557.

[29] GramVaani. 2018. About us. http://www.gramvaani.org/. [Online; accessed 2-Jan-2021].

[30] Xinning Gui, Yu Chen, Yubo Kou, Katie Pine, and Yunan Chen. 2017. Investigating support seeking from peers for pregnancy in online health communities. Proceedings of the ACM on Human-Computer Interaction 1, CSCW (2017), 1-19.

[31] Andrea L Hartzler, Leah Tuzzio, Clarissa Hsu, and Edward H Wagner. 2018. Roles and functions of community health workers in primary care. The Annals of Family Medicine 16, 3 (2018), 240-245.

[32] UNICEF India. 2019. Early childhood nutrition. https://www.unicef.org/india/ what-we-do/early-childhood-nutrition. [Online; accessed 11-Jan-2021]

[33] IntraHealth International. 2015. Use of an Interactive Voice Response System to Deliver Refresher Training in Senegal. https://www.capacityplus.org/files/ resources/ivr-system-refresher-training-senegal.pdf.

[34] Azra Ismail and Neha Kumar. 2018. Engaging solidarity in data collection practices for community health. Proceedings of the ACM on Human-Computer Interaction 2, CSCW (2018), 1-24.

[35] Azra Ismail and Neha Kumar. 2019. Empowerment on the margins: The online experiences of community health workers. In Proceedings of the $2019 \mathrm{CHI}$ Conference on Human Factors in Computing Systems. 1-15.

[36] Maham Javaid, Beenish Fatima, and Amna Batool. 2017. Bridging the Knowledge Gaps in Lady Health Visitors through Video Based Learning Tool. In Proceedings of the Ninth International Conference on Information and Communication
Technologies and Development. ACM, 38 .

[37] T Jacob John, Lalit Dandona, Vinod P Sharma, and Manish Kakkar. 2011. Continuing challenge of infectious diseases in India. The Lancet 377, 9761 (2011), $252-269$

[38] Gareth Jones, Richard W Steketee, Robert E Black, Zulfiqar A Bhutta, Saul S Morris, Bellagio Child Survival Study Group, et al. 2003. How many child deaths can we prevent this year? The lancet 362, 9377 (2003), 65-71.

[39] Matthew Kam, Anuj Kumar, Shirley Jain, Akhil Mathur, and John Canny. 2009. Improving literacy in rural India: Cellphone games in an after-school program. In 2009 International Conference on Information and Communication Technologies and Development (ICTD). IEEE, 139-149.

[40] Justine A Kavle, Elizabeth LaCroix, Hallie Dau, and Cyril Engmann. 2017. Addressing barriers to exclusive breast-feeding in low-and middle-income countries: a systematic review and programmatic implications. Public Health Nutrition 20, 17 (2017), 3120-3134.

[41] Konstantinos Kazakos, Siddhartha Asthana, Madeline Balaam, Mona Duggal, Amey Holden, Limalemla Jamir, Nanda Kishore Kannuri, Saurabh Kumar, Amarendar Reddy Manindla, Subhashini Arcot Manikam, et al. 2016. A real-time ivr platform for community radio. In Proceedings of the 2016 CHI Conference on Human Factors in Computing Systems. 343-354.

[42] Maryse C Kok, Marjolein Dieleman, Miriam Taegtmeyer, Jacqueline EW Broerse, Sumit S Kane, Hermen Ormel, Mandy M Tijm, and Korrie AM de Koning. 2015. Which intervention design factors influence performance of community health workers in low-and middle-income countries? A systematic review. Health policy and planning 30, 9 (2015), 1207-1227.

[43] Maryam Eslahcar Komachali and Mohammadreza Khodareza. 2012. The Effect of Using Vocabulary Flash Card on Iranian Pre-University Students' Vocabulary Knowledge. International Education Studies 5, 3 (2012), 134-147.

[44] Kenneth L Kraemer, Jason Dedrick, and Prakul Sharma. 2009. One laptop per child: vision vs. reality. Commun. ACM 52, 6 (2009), 66-73.

[45] Arun Kumar, Nitendra Rajput, Dipanjan Chakraborty, Sheetal K Agarwal, and Amit A Nanavati. 2007. WWTW: the world wide telecom web. In Proceedings of the 2007 workshop on Networked systems for developing regions. 1-6.

[46] Anuj Kumar, Pooja Reddy, Anuj Tewari, Rajat Agrawal, and Matthew Kam. 2012. Improving literacy in developing countries using speech recognition-supported games on mobile devices. In Proceedings of the SIGCHI Conference on Human Factors in Computing Systems. 1149-1158.

[47] Neha Kumar, Susan M Dray, Christian Sturm, Nithya Sambasivan, Laura S Gaytán-Lugo, Leonel V Morales Diaz, Negin Dahya, and Nova Ahmed. 2017. HCI Across Borders. In Proceedings of the 2017 CHI Conference Extended Abstracts on Human Factors in Computing Systems. 3065-3072.

[48] Neha Kumar, Trevor Perrier, Michelle Desmond, Kiersten Israel-Ballard, Vikrant Kumar, Sudip Mahapatra, Anil Mishra, Shreya Agarwal, Rikin Gandhi, Pallavi Lal, et al. 2015. Projecting health: community-led video education for maternal health. In Proceedings of the Seventh International Conference on Information and Communication Technologies and Development. ACM, 17.

[49] Simon Lewin, Susan Munabi-Babigumira, Claire Glenton, Karen Daniels, Xavier Bosch-Capblanch, Brian E Van Wyk, Jan Odgaard-Jensen, Marit Johansen, Godwin N Aja, Merrick Zwarenstein, et al. 2010. Lay health workers in primary and community health care for maternal and child health and the management of infectious diseases. Cochrane database of systematic reviews 3 (2010).

[50] Nancy K Lowe. 2011. The Surgeon General's call to action to support breastfeeding. Fournal of Obstetric, Gynecologic \& Neonatal Nursing 40, 4 (2011), 387-389.

[51] Michael A Madaio, Evelyn Yarzebinski, Vikram Kamath, Benjamin D Zinszer, Joelle Hannon-Cropp, Fabrice Tanoh, Yapo Hermann Akpe, Axel Blahoua Seri, Kaja K Jasińska, and Amy Ogan. 2020. Collective Support and Independent Learning with a Voice-Based Literacy Technology in Rural Communities. In Proceedings of the 2020 CHI Conference on Human Factors in Computing Systems. $1-14$.

[52] Robert McPherson and Stephen Hodgins. 2018. Postnatal home visitation: lessons from country programs operating at scale. Journal of global health 8,1 (2018).

[53] Indrani Medhi, Raghu S Menon, Edward Cutrell, and Kentaro Toyama. 2012. Correlation between limited education and transfer of learning. Information Technologies \& International Development 8, 2 (2012), pp-51.

[54] Indrani Medhi, Somani Patnaik, Emma Brunskill, SN Nagasena Gautama, William Thies, and Kentaro Toyama. 2011. Designing mobile interfaces for novice and low-literacy users. ACM Transactions on Computer-Human Interaction (TOCHI) 18, 1 (2011), 1-28.

[55] National Health Mission. 2019. Guidelines for Community Processes. https://nhm.gov.in/images/pdf/communitisation/asha/OrdersGuidelines/Guidelines_for_Community_Processes_2014_English.pdf. [Online; accessed 11-Jan-2021].

[56] National Health Mission. 2019. Quarterly NHM MIS Report. https://nhm.gov.in/. [Online; accessed 5-May-2020].

[57] National Health Mission. 2021. About us. http://nhm.gov.in/. [Online; accessed 11-Jan-2021] 
[58] Sugata Mitra, Ritu Dangwal, Shiffon Chatterjee, Swati Jha, Ravinder S Bisht, and Preeti Kapur. 2005. Acquisition of computing literacy on shared public computers: Children and the "hole-in-the-wall". Australasian fournal of Educational Technology 21, 3 (2005)

[59] Aparna Moitra, Vishnupriya Das, Gram Vaani, Archna Kumar, and Aaditeshwar Seth. 2016. Design lessons from creating a mobile-based community media platform in Rural India. In Proceedings of the Eighth International Conference on Information and Communication Technologies and Development. 1-11.

[60] Maletsabisa Molapo, Melissa Densmore, and Brian DeRenzi. 2017. Video Consumption Patterns for First Time Smartphone Users: Community Health Workers in Lesotho. In Proceedings of the 2017 CHI Conference on Human Factors in Computing Systems. ACM, 6159-6170.

[61] Maletsabisa Molapo and Gary Marsden. 2013. Software support for creating dig ital health training materials in the field. In Proceedings of the Sixth International Conference on Information and Communication Technologies and Development: Full Papers-Volume 1. ACM, 205-214.

[62] Thomas R Moore. 1997. Clinical assessment of amniotic fluid. Clinical obstetrics and gynecology 40, 2 (1997), 303-313.

[63] Preeti Mudliar, Jonathan Donner, and William Thies. 2013. Emergent practices around CGNet Swara: A voice forum for citizen journalism in rural India. Information Technologies \& International Development 9, 2 (2013), pp-65.

[64] Maryam Mustafa, Amna Batool, Beenish Fatima, Fareeda Nawaz, Kentaro Toyama, and Agha Ali Raza. 2020. Patriarchy, Maternal Health and Spiritual Healing: Designing Maternal Health Interventions in Pakistan. In Proceedings of the 2020 CHI Conference on Human Factors in Computing Systems. 1-13.

[65] NHSRC. 2019. Update on ASHA Programme, January 2019. http://nhsrcindia. org/.

[66] Ministry of Health and Govt. of India Family Welfare. 2006. Book 2. https: //nhm.gov.in/index1.php?lang=1\&level=3\&sublinkid=184\&lid=257. [Online; accessed 5-May-2020].

[67] Ministry of Health and Govt. of India Family Welfare. 2006. Book 3. https: $/ /$ nhm.gov.in/index1.php?lang=1\&level=3\&sublinkid=184\&lid=257. [Online; accessed 5-May-2020].

[68] Ministry of Health and Govt. of India Family Welfare. 2006. Book 6. https: $/ /$ nhm.gov.in $/$ index1.php?lang=1\&level=3\&sublinkid=184\&lid=257. [Online; accessed 5-May-2020].

[69] Ministry of Health and Govt. of India Family Welfare. 2006. Book 7. https $/ /$ nhm.gov.in/index1.php?lang=1\&level=3\&sublinkid=184\&lid=257. [Online accessed 5-May-2020].

[70] Ministry of Home Affairs. 2011. Rural Urban Distribution of Population. https: //censusindia.gov.in/. [Online; accessed 16-Sep-2020].

[71] Times of India. 2020. ASHA workers 60,000, Maharashtra to buy 2,070 smart phones. https://timesofindia.indiatimes.com/city/nagpur/ashaworkers-60k-maha-to-buy-2070-smart-phones/articleshow/68118360.cms\#: :text=NAGPUR\%3A\%20State\%20health\%20department\%20has,(ASHA) \%20workers\%20in\%20Maharashtra.. [Online; accessed 16-Sep-2020].

[72] Government of India Ministry of Health and Family Welfare Statistics Division. 2015. Rural Health Statistics 2014-2015. https://wcd.nic.in/. [Online; accessed 07-Feb-2020].

[73] International Institute of Population Sciences. 2016. National Family Health Survey-4. http://rchiips.org/. [Online; accessed 25-May-2020]

[74] World Health Organization. 2010. World Health Statistics 2019, Monitoring Health for the SDGs. https://www.who.int/gho/publications/world_health_ statistics/2019/en/. [Online; accessed 07-Feb-2020].

[75] World Health Organization. 2014. Integrated Management of Childhood Illness: distance learning course. World Health Organization. 15 booklets pages.

[76] World Health Organization. 2019. Exclusive breastfeeding for optimal growth development and health of infants. https:/www.who.int/elena/titles/exclusive breastfeeding/en/. [Online; accessed 1-Apr-2019].

[77] World Health Organization. 2020. Medical doctors (per 10000 population). https://www.who.int/data/gho/data/indicators/indicator-details/GHO/ medical-doctors-(per-10-000-population). [Online; accessed 12-Jan-2021]

[78] World Health Organization et al. 2007. Community health workers: What do we know about them. Geneva: WHO Department of Human Resources for Health (2007).

[79] World Health Organization et al. 2018. Implementation guidance: protecting, promoting and supporting breastfeeding in facilities providing maternity and newborn services: the revised baby-friendly hospital initiative. (2018).

[80] Outlook. 2020. Himachal: ASHA workers get smartphones to perform duties efficiently. https://www.outlookindia.com/newsscroll/himachal-asha-workersget-smartphones-to-perform-duties-efficiently/1896227. [Online; accessed 16-Sep-2020].

[81] Niranjan Pai, Pradnya Supe, Shailesh Kore, YS Nandanwar, Aparna Hegde, Edward Cutrell, and William Thies. 2013. Using automated voice calls to improve adherence to iron supplements during pregnancy: a pilot study. In Proceedings of the Sixth International Conference on Information and Communication Technologies and Development: Full Papers-Volume 1. 153-163.
[82] Joyojeet Pal, Anjuli Dasika, Ahmad Hasan, Jackie Wolf, Nick Reid, Vaishnav Kameswaran, Purva Yardi, Allyson Mackay, Abram Wagner, Bhramar Mukherjee, et al. 2017. Changing data practices for community health workers: Introducing digital data collection in West Bengal, India. In Proceedings of the Ninth International Conference on Information and Communication Technologies and Development. 1-12.

[83] Tapan S Parikh, Paul Javid, Kaushik Ghosh, and Kentaro Toyama. 2006. Mobile phones and paper documents: evaluating a new approach for capturing microfinance data in rural India. In Proceedings of the SIGCHI conference on Human Factors in computing systems. 551-560.

[84] Neil Patel, Deepti Chittamuru, Anupam Jain, Paresh Dave, and Tapan S Parikh. 2010. Avaaj otalo: a field study of an interactive voice forum for small farmers in rural india. In Proceedings of the SIGCHI Conference on Human Factors in Computing Systems. ACM, 733-742.

[85] Trevor Perrier, Nicola Dell, Brian DeRenzi, Richard Anderson, John Kinuthia, Jennifer Unger, and Grace John-Stewart. 2015. Engaging pregnant women in Kenya with a hybrid computer-human SMS communication system. In Proceedings of the 33rd Annual ACM Conference on Human Factors in Computing Systems. $1429-1438$

[86] Anthony Poon, Sarah Giroux, Parfait Eloundou-Enyegue, François Guimbretière, and Nicola Dell. 2019. Engaging high school students in cameroon with exam practice quizzes via sms and whatsapp. In Proceedings of the 2019 CHI Conference on Human Factors in Computing Systems. 1-13.

[87] Divya Ramachandran, John Canny, Prabhu Dutta Das, and Edward Cutrell. 2010. Mobile-izing health workers in rural India. In Proceedings of the SIGCHI Conference on Human Factors in Computing Systems. ACM, 1889-1898.

[88] Emily Rhodes and Greg Walsh. 2016. Recommendations for developing technologies that encourage reading practices among children in families with low-literate adults. In Proceedings of the The 15th International Conference on Interaction Design and Children. 125-136.

[89] Heather J Rowe-Murray and Jane RW Fisher. 2002. Baby friendly hospital practices: cesarean section is a persistent barrier to early initiation of breastfeeding. Birth 29, 2 (2002), 124-131.

[90] Marie-Josèphe Saurel-Cubizolles, Patrizia Romito, Nathalie Lelong, and PierreYves Ancel. 2000. Women's health after childbirth: a longitudinal study in France and Italy. BfOG: An International fournal of Obstetrics \& Gynaecology 107, 10 (2000), 1202-1209.

[91] Amit Saxena, Urvashi Sahni, Rahul Gupta, Anjana Arora, Richard Anderson, and Natalie Linnell. 2010. Evaluating facilitated video instruction for primary schools in rural india. In Proceedings of the 4th ACM/IEEE International Conference on Information and Communication Technologies and Development. 1-10.

[92] David K Stevenson, Phyllis A Dennery, and Susan R Hintz. 2001. Understanding Newborn Jaundice<!--. Journal of Perinatology 21, S1 (2001), S21-S21.

[93] Kentaro Toyama. 2011. There Are No Technology Shortcuts to Good Education. http://edutechdebate.org/ict-in-schools/there-are-no-technologyshortcuts-to-good-education/.

[94] Kentaro Toyama. 2015. Geek Heresy: Rescuing Social Change from the Cult of Technology. Perseus Books Group.

[95] TulaSalud. 2020. About us. http://www.tulasalud.org/. [Online; accessed 22-Dec-2020]

[96] Anupriya Tuli, Shaan Chopra, Neha Kumar, and Pushpendra Singh. 2018. Learning from and with Menstrupedia: Towards menstrual health education in India. Proceedings of the ACM on Human-Computer Interaction 2, CSCW (2018), 1-20.

[97] Elba Del Carmen Valderrama Bahamóndez, Bastian Pfleging, Niels Henze, and Albrecht Schmidt. 2014. A long-term field study on the adoption of smartphones by children in panama. In Proceedings of the 16th international conference on Human-computer interaction with mobile devices \& services. 163-172.

[98] Elba del Carmen Valderrama Bahamondez, Christian Winkler, and Albrech Schmidt. 2011. Utilizing multimedia capabilities of mobile phones to support teaching in schools in rural panama. In Proceedings of the SIGCHI Conference on Human Factors in Computing Systems. 935-944.

[99] Rama Adithya Varanasi, René F Kizilcec, and Nicola Dell. 2019. How Teachers in India Reconfigure their Work Practices around a Teacher-Oriented Technology Intervention. Proceedings of the ACM on Human-Computer Interaction 3, CSCW (2019), 1-21.

[100] Rama Adithya Varanasi, Aditya Vashistha, Tapan Parikh, and Nicola Dell. 2020. Challenges and Issues Integrating Smartphones into Teacher Support Programs in India. In Proceedings of the 2020 International Conference on Information and Communication Technologies and Development. 1-11.

[101] Aditya Vashistha, Edward Cutrell, Gaetano Borriello, and William Thies. 2015. Sangeet swara: A community-moderated voice forum in rural india. In Proceedings of the 33rd Annual ACM Conference on Human Factors in Computing Systems. ACM, 417-426.

[102] Aditya Vishwanath, Matthew Kam, and Neha Kumar. 2017. Examining low-cost virtual reality for learning in low-resource environments. In Proceedings of the 2017 Conference on Designing Interactive Systems. 1277-1281.

[103] UNAIDS WHO et al. 2008. Task shifting: global recommendations and guidelines. WHO 94 (2008), 407 
[104] Joseph Jay Williams, Tania Lombrozo, Anne Hsu, Bernd Huber, and Juho Kim. 2016. Revising learner misconceptions without feedback: Prompting for reflec tion on anomalies. In Proceedings of the 2016 CHI Conference on Human Factors in Computing Systems. 470-474.

[105] Leslie Owen Wilson. 2016. Anderson and Krathwohl-Bloom's taxonomy revised. Understanding the New Version of Bloom's Taxonomy (2016).

[106] Deepika Yadav, Anushka Bhandari, and Pushpendra Singh. 2019. LEAP: Scaffolding Collaborative Learning of Community Health Workers in India. Proceedings of the ACM on Human-Computer Interaction 3, CSCW (2019), 1-27.

[107] Deepika Yadav, Mayank Gupta, Malolan Chetlur, and Pushpendra Singh. 2018 Automatic Annotation of Voice Forum Content for Rural Users and Evaluation of Relevance. In Proceedings of the 1st ACM SIGCAS Conference on Computing and Sustainable Societies. ACM, 12.

[108] Deepika Yadav, Prerna Malik, Kirti Dabas, and Pushpendra Singh. 2019. Feedpal Understanding opportunities for chatbots in breastfeeding education of women in india. Proceedings of the ACM on Human-Computer Interaction 3, CSCW
(2019), 1-30.

[109] Deepika Yadav, Pushpendra Singh, Kyle Montague, Vijay Kumar, Deepak Sood, Madeline Balaam, Drishti Sharma, Mona Duggal, Tom Bartindale, Delvin Varghese, et al. 2017. Sangoshthi: Empowering Community Health Workers through Peer Learning in Rural India. In Proceedings of the 26th International Conference on World Wide Web. International World Wide Web Conferences Steering Committee, 499-508.

[110] Juan Yang, Katherine E. Hartmann, David A. Savitz, Amy H. Herring, Nancy Dole, Andrew F. Olshan, and Jr. Thorp, John M. 2004. Vaginal Bleeding during Pregnancy and Preterm Birth. American fournal of Epidemiology 160, 2 (07 2004), $118-125$.

[111] Dejan Zurovac, Raymond K Sudoi, Willis S Akhwale, Moses Ndiritu, Davidson H Hamer, Alexander K Rowe, and Robert W Snow. 2011. The effect of mobile phone text-message reminders on Kenyan health workers' adherence to malaria treatment guidelines: a cluster randomised trial. The Lancet 378, 9793 (2011), 795-803. 